\title{
Various types of echelette resonators for gyrotrons
}

\author{
V.E.Zapevalov, S.N.Vlasov, E.V.Koposova, A.N.Kuftin, A.B.Paveliev, N.A.Zavolsky
}

Federal Research Center Institute of Applied Physics, RAS, Nizhny Novgorod, Russia, zapev@appl.sci-nnov.ru,

In recent years, significant progress has been made in the development of high-performance gyrotrons and various applications based on them $[1,2]$. In the gyrotrons of the millimeter wavelength range the resonators (cavities) in the form of waveguides of variable cross section are used. In the operating regime, they radiate into the output waveguide one rotating operating $\mathrm{TE}_{\mathrm{mp}}$ mode with azimuthal $m$ and radial $p$ indices. These resonators have the so-called minimum diffraction Q-factor, determined by the ratio of the resonator length to the wavelength of the radiation. The existence of this quantity is due to the operation of the resonator at the quasicritical frequency of the working mode and the presence of a "diffractive" single-mode output from the resonator. The existence of a lower limit of the diffraction Q-factor (at a finite value of the ohmic Q-factor) limits the power that can be obtained from a gyrotron with a given mode.The increase in power is achieved by increasing the surface area of the resonator, for which it is necessary to increase its cross section. However, this is possible up to a certain limit because of the thickening of the spectrum of eigen frequencies of the resonator and the deterioration of the mode selection. Thus, the possibilities of increasing the output power and operating frequency are limited, especially in gyrotrons working on gyrofrequency harmonics. Overcoming this limitation is possible with the use of resonators in which the minimum value of the diffraction $\mathrm{Q}$-factor is not limited so rigidly by their length. In this paper the results of a study of several variants of gyrotrons with echelette-type resonators are presented.

Echelette resonator is one of the possible resonators with the required properties. In comparison with conventional resonators, the power flux density to the wall here can be reduced severalfold. The principles of constructing echelette resonators are described in [3-6]. In such resonators the operating modes are formed by reflection of waves from corrugated surfaces in an autocollimation regime. Three types of echelette resonators are discussed: a) two-mirror resonator, in which the field is represented as waves propagating towards each other, b) three-mirror resonator, in which only one mirror is corrugated, and the field represents a wave traveling along the azimuth, c) an axially symmetric resonator with a corrugated surface. Resonators a) and c) are investigated in this paper in more detail. The electron beam is assumed to be axially symmetric, which has by now been well developed. However, in some cases, in particular for a two-mirror circuit, it is advisable to use ribbon beams to increase the efficiency and additional selection.

In Fig. 1 the profile of the axially symmetric echelette resonator, RF field distribution (a), and the photo of this resonator (b) are presented. The resonator has sinusoidal corrugation and was designed to operate at the 1st harmonic of the gyrofrequency and a symmetrical operating mode.

The operating frequency of the gyrotron is $150 \mathrm{GHz}$ and the accelerating voltage $-80 \mathrm{kV}$. With the chosen parameters of the resonator and the electron magnetron-injector gun described in [7], the theoretical value of $Q \approx 4200$ provides the achievement of the maximum value of electron efficiency.

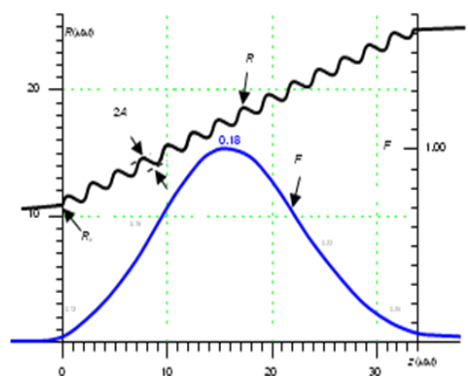

(a)

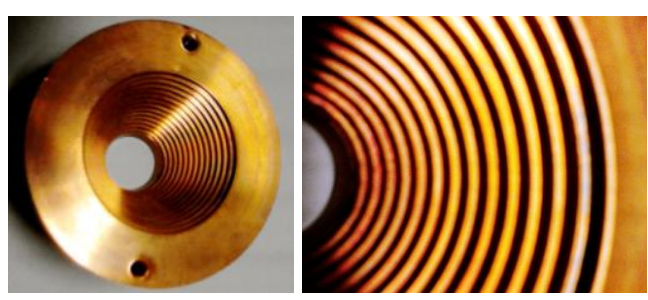

(b)

Fig. 1. The profile of the echeltte resonator and the distribution of the high-frequency field (a) and the photos of the resonator and its part (b)

An experimental study of the gyrotron was carried out according to the routine scheme using the apparatus described in [8]. The dependences of the pulse output power $P$ (pulse duration $\tau=50 \mu \mathrm{s}$ at repetition frequency $f_{0}=5 \mathrm{~Hz}$ ) on the magnetic induction in cryomagnet $B_{0}$ (current in the solenoid) were obtained at a voltage $U=80 \mathrm{kV}$ for different values of the electron beam current $I_{b}$ taken from cathode (see Fig. 2).

The experimental data demonstrates only one oscillation in the magnetic field tuning band. A high value of the output efficiency $\eta \approx 32 \%$ took place at the current of the electron beam $I_{b}=35 \mathrm{~A}$. Thus, the axially symmetric echelette gyrotron has a much more sparse spectrum of eigenfrequencies and a noticeably lower specific thermal load on the resonator walls compared with the canonical gyrotron (1.8 times) with a close efficiency. The decrease in the thermal load is due to both a decrease in the quality factor in comparison with a cylindrical resonator of similar length and a certain increase in the surface. 


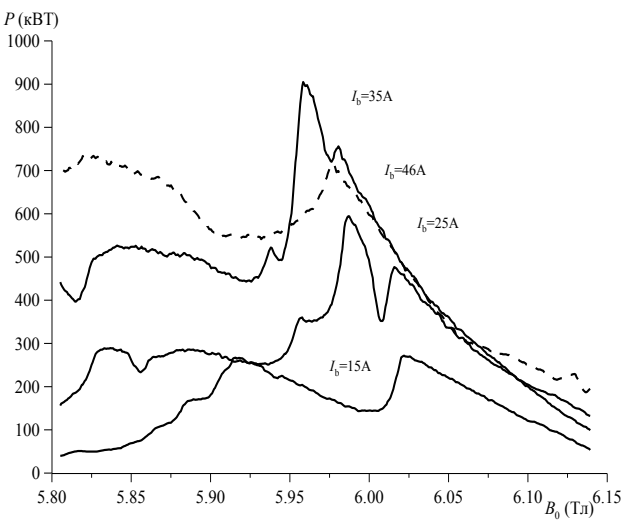

Fig. 2. Dependence of the output power of the gyrotron on the magnetic field in the resonator for different values of the electron beam current

For a number of applications a two-mirror version of the echelette gyrotron is interested. In this variant, there are some possibilities for frequency tuning and the radiation output system is simplified but with the use of a cylindrical beam an efficiency decrease of 1.5-2 times is inevitable. This scheme is the development of gyrotrons with a quasioptical resonator with additional selection due to the echelette mirror. Fig. 3 shows a gyrotron in which a two-mirror echelette resonator is used. Here the RF field can be represented in the form of waves propagating towards each other.

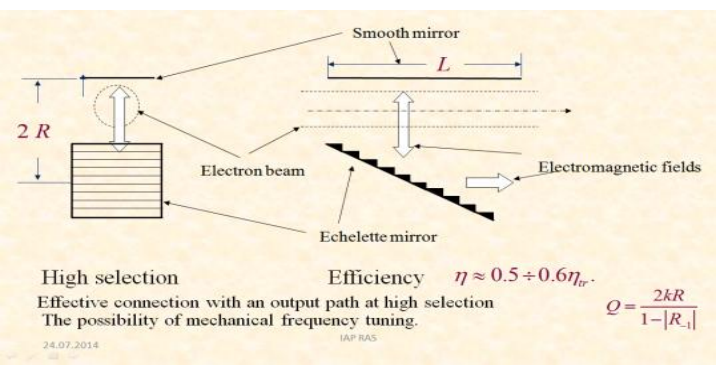

Fig. 3 Scheme of the gyrotron with a two-mirror echelette resonator

Calculations carried out for gyrotrons with twomirror echelette resonators operating at the third harmonic of the gyrofrequency demonstrate the promises of these systems. Estimations of the $Q$-factor of resonators and start-up regimes for these gyrotrons were performed. The electron beam was assumed to be axially symmetric and had the same design parameters as indicated above. The possibilities of smooth tuning of the frequency in such systems were investigated. Calculations show that the use of two-mirror echelette resonators in gyrotrons operating at the $1 \mathrm{st}$ to 3rd harmonics of the gyrofrequency makes it possible to realize a smooth tuning of their operating frequency in the band of about $1 \%$ by varying the distance between the mirrors and corresponding correction of the magnetic field.

To increase the efficiency and additional selection in a two-mirror scheme it is expedient to use ribbon beams. For the same purposes, it is also possible to develop cylindrical beams with axially inhomogeneous emission. The development of electron-optical systems for the formation of such beams has its own specifics and is in the initial stage.

A three-mirror echelette resonator consisting of one echelette (corrugated) and two smooth mirrors possesses all the advantages of a two-mirror resonator exceeding its efficiency in the use of an electron beam of a cylindrical configuration.

Theoretical and experimental studies have shown that the axially symmetric echelette gyrotron has a significantly more rare spectrum of eigenfrequencies and a lower thermal load (by a factor of 1.8) on the resonator walls compared to the canonical gyrotron with close efficiencies. This allows us to hope for obtaining in the echelette gyrotrons more power at long-pulsed and continuous regimes. The output radiation in the form of a conical wavebeam can be converted, for example, into the $\mathrm{TE}_{01}$ wave of a waveguide of circular cross section.

The performed analytical estimations and results of numerical simulation show the possibility to realize the smooth frequency tuning within the band of about $1 \%$ in the gyrotrons with two-mirror echelette resonators operating on the 1-3th harmonics of the gyrofrequency.

Echelette gyrotrons have great prospects, but in their calculation and manufacturing technology serious problems are encountered. A complex structure of the output radiation requires the development of new variants of the converter.

\section{Acknowledgements}

The work was supported by the Russian Foundation for Basic Research (Grant 18-02-00832 A).

\section{References}

1. Nusinovich G.S., Thumm M., Petelin M.I. The Gyrotron at 50: Historical Overview. // J. Infrared $\mathrm{Mm} \mathrm{THz}$ Waves. 2014. V. 35, No. 4, P. 325-381.

2. Zapevalov V.E. Evolution of gyrotrons // Radiophysics and Quantum electronics. 2011. V. LIV, No. 8-9, P. 559572 .

3. Kosarev E.L. Resolution of the Spectrum of an Open Resonator with the Aid of an Echelette Grating // JETP Letters. 1966. V. 3, No. 7, P. 190-192.

4. Vlasov S.N., Koposova E.V., Pavel'ev A.B., Khizhnyak V.I. Gyrotrons with echelette resonators // Radiophysics and Quantum Electronics. 1996. V. 39, No. 6, P. 458462 .

5. Vlasov S.N., Zavolsky N.A., Zapevalov V.E., Koposova E.V., Moiseev M.A. Axisymmetric multistage cavity resonators // Radiophysics and Quantum electronics. 2009. V. 52, No. 9, P. 642-654.

6. Vlasov S.N., Koposova E.V. Traveling-wave echelette cavity // Radiophysics and Quantum electronics. 2014. V. 56, No. 10, P. 658-663.

7. Zapevalov V.E, Kuftin AN, Lygin V.K. Numerical simulation and experimental study of an electron-optical system of a megawatt gyrotron with step frequency tuning in the range 100-170 GHz. 2007. V. 50, No. 9, P. 702-712.

8. Zapevalov V.E., Bogdashov A.A., G.G. Denisov, Kuftin A.N., Lygin V.K., Moiseev M.A., Chirkov A.V. Development of a prototype of a 1-MW 105-156-GHz multifrequency gyrotrons gyrotrons // Radiophysics and Quantum electronics. 2004. V. 47, No. 5-6, P. 396-404. 